\title{
Effect of Some Macro Elements and Bio Fertilization on The Growth, Yield and Chemical Composition of Coriander Plant (Coriandrum sativum L.)
}

\author{
${ }^{1}$ Nasr Alla, M. W., ${ }^{1}$ F. I., Radwan, ${ }^{1}$ A.A. Abido, ${ }^{2}$ S.H. Shaban \\ ${ }^{1}$ Plant Production Dept., Faculty of Agriculture (Saba Basha), Alexandria University \\ ${ }^{2}$ Medicinal and Aromatic Res.Dept., A.R.C. Alexandria, Egypt
}

\begin{abstract}
Coriander (Coriandrum sativum, L.) is an important medicinal herbs worldaride. The objective of this study was to investigate the effect of mineral and bio fertilization on vegetative growth, yield, and chemical composition including essential oil; as well as, major compounds of Coriandrum sativum L. Therefore, two filed experiments were conducted at the Experimental Farm of the Nubaria station-ELbeharia, Egypt during 2013/2014 and 2014/2015 growing seasons. The experimental design was split plot design with three replicates. Mineral fertilizer levels (control, 50\% NPK, 75\% NPK and 100\% NPK) occupied the main plots. The sub plot was assigned to five bio-fertilizer treatments, i.e.,uninoculation, phosphorein, cerealine, potassomage and mixed bio-fertilizer). Theresultsobtained could be summarized as follows: (1) the addition of mineral fertilizer at rate of $100 \%$ resulted in a significant increment in vegetative growth,yield and chemical composition of coriander plants during both seasons, (2) the application of $100 \%$ NPK with mixture of biofertilizers (phosphorein +cerealine + potasisomage) was the best combination to obtain the highest mean values of plant height, number of branches/plant, fresh and dry weights of herb/plant, number of umbels/plant, 100-seed weight and seed yield/fed, as well as, oil \%, but major compounds resulted fromcombination of $75 \%$ NPK with potassomage and mix of biofertilizers for the coriander were tested in this study. However, of traits under study increased significantly, due to inoculation treatments over the uninoculation (control) one and the mixture of biofertilizers was the best treatment. This investigation suggests the need for more studies concerning the effect of NPK fertilizer rates and biofertilizers on coriander plants under different environments using different types of soil in order to reach the optimum combination to achieve the best yield.

Keywords: Mineral fertilizers, biofertilization, coriander major compounds.
\end{abstract}

\section{INTRODUCTION}

Coriandrum sativum L. (coriander)Fam.Apiaceae is an important fresh culinary herb in the United States, widely used in Mexican, African and Asian cuisines (Cantwell and Reid, 1993). It is used as a spice in a wide variety of foods. The pungent leaves are called "cilantro" a basic ingredient in Latin American and Asian cooking. The leaves (cilantro) have a bold taste, combining a strong sage flavor with sharp-citrus noted. The roots are similar with an added nutty flavor and are used fresh only. The seeds of coriander have a taste of citrus and are used in medicinal house hold cosmetics and fragrance (Rashed, 2002).

Fertilization is one of the most important factors limiting the productivity of plants. The intensive use of expensive mineral fertilizers results in environmental pollution problems. Further, chemical fertilizers at extremely high rates to a long period decreased the potential activity of microflora and the stability of soil organic matter (Hussien, 1995).Also, bio-fertilization is an important factor being used to produce products without some mineral fertilizer that cause environmental pollution problems. Hence, the attention has been focused on the recreates of biofertilizers to safe alternative specific chemical fertilizers. Biofertilizers play a vital role for increasing the number of 
microorganisms and accelerate certain microbial process in the rhizosphere of inoculated soil of plants (Kandeel et al., 2001;Rashed, 2002; Mohamed and Abdu, 2004). This research, however, is an attempt to find out the best fertilization treatments (mineral NPK and biofertilizer on the vegetative growth, yield and chemical composition of corianderplants (Coriandrum sativum L.).

\section{MATERIALS AND METHODS}

Two filed experiments were carried out at the Experimental Farm of the Nubaria station, El-beharia, Egypt during both seasons of2013/2014 and 2014/2015, to study the effect of NPK, biofertilizer and their interaction on the growth,yield, chemical composition of coriander plants(Coriandrum sativum L.). The experimental design followed in this work was a split plot design with three replicates.where as the chemical fertilizers were arranged as maim plots and biofertilizers were arranged as sub plots. The replicate contain 20 treatments. The coriander seeds were sown on November $11^{\text {th }}$ and $12^{\text {th }}$ during both growing seasons. Each sub plot in both experimental seasons was 3.5 meters long and 3 meter wide $\left(10.5 \mathrm{~m}^{2}\right.$ i.e. $1 / 400$ feddan) contained 10 rows $25 \mathrm{~cm}$ apart between plants. The applied chemical fertilizer were ammonium sulphate $(20.5 \% \mathrm{~N})$, calcium superphosphate $\left(15.5 \% \mathrm{P}_{2} \mathrm{O}_{5}\right)$ and potassium sulphate $(48 \%$ $\left.\mathrm{K}_{2} \mathrm{O}\right)$ at the rates of $(100,100$ and $50 \mathrm{~kg} / \mathrm{fed})$, respectively which are the recommended dose. The used biofertilization of bacteria was phosphorein (Bacillus megatherium phosphorus dissoliving bacteria P. D.B.), potassiomage biofertilizer contains of Bacillus mucilaginousas a potassium dissolving bacteria (KDB) or potassium solubilizing bacteria (KSB) and cerealine (Azosprillum lipoferum and Azotobacter chroococcum) which supplied by Natioal Research Center. The inoculation with phosphorein, potassiomage and cerealine were performed by coating coriander seed with each product individually using a sticking substance (Arbic gum at 5\%) just before sowing. The recommended dose of NPK was divided into two equal parts, the first one was applied one month after sowing and the second one was applied before the first irrigation. The tested treatments were conducted as follows:

A) NPK fertilizer (Main plot)

- Control ( without fertilization )

- $50 \%$ NPK

- $75 \% \mathrm{NPK}$

- $100 \%$ NPK

B) Biofertilization (sub plot)

- Uninoculation (control)

- Phosphorein

- Cerealine

- Potassiomage

- Mixture (phosphorein + cerealine + potassiomage)

The physical and chemical characteristics of the experimental soil are given in Table (1). The soil was analyzed according to the methods described by Page et al. (1982). At harvest dates on April $8^{\text {th }}$ and $10^{\text {th }}$ during the two seasons, guarded plants were randomly taken from each plot and the following characteristics were recorded: 
1. Plant height $(\mathrm{cm})$

2. Number of branches

3. Fresh and dry weight $(\mathrm{g}) /$ plant

4. Number of umbles/plant

5. 100-seed weight $(\mathrm{g})$

6. Seed yield $(\mathrm{g}) /$ plant

7. Seed yield (ton/fed)

8. Total chlorophyll ( $\mathrm{mg} / \mathrm{g}$ fresh weight)was determined in fresh leaves, samples of the fifth leaf from top at harvesting using the methods by Moran (1982).

9. The $\mathrm{N}, \mathrm{P}$ and $\mathrm{K}$ contents were determined in the leaves which were driedat $70^{\circ} \mathrm{C}$ for $48 \mathrm{hr}$., and ground leaves $(0.5 \mathrm{~g})$ were digested with sulphuric acid and hydrogen peroxide $\mathrm{H}_{2} \mathrm{SO}_{4} . \mathrm{H}_{2} \mathrm{O}_{2}$ according to the method of Lowther (1980) and the following determination were carried out in the digested solution to determine the following:

- Nitrogen content (N\%)

Nitrogen was determined in the digested plant material colorimetrically by Nessler`smethod(Chapman and Pratt, 1978).

- Phosphorus content (P \%)

Phosphorus was determined by the Vanadomolyate yellow methodas given by Jackson (1973) and the intensity of color developed was read by spectrophotometer at $405 \mathrm{~nm}$.

- Potassium content ( $\mathrm{K} \%$ )

Potassium was determined according to the method described by Jackson (1973) using Beckman Flame photometer.

- Essential oil percentage was determined in the air-dried seeds according to British Pharmacopocia (1963) by water distillation $40 \mathrm{~g}$ of herb for 1.5-2 hour, in order to extract the essential oil.

- The percentage of major compounds (linolool, $\alpha$ - 2-deenol, $\alpha$ pinene,camphor and geraniol) were estimated by measuring the peak area of the different compounds of the chromatogram according to Heftman (1967) and Gunther and Joseph (1978).

The obtained data were analyzed statistically for ANOVA and L.S.D. values were calculated to least differences between the studied treatments according to Gomez and Gomez (1984). 
Table (1). Physical and chemical analysis of the used soil before planting (average of 2013/2014 and 2014/2015seasons.

\begin{tabular}{|c|c|c|}
\hline Parameter & Sample & Unit \\
\hline \multicolumn{3}{|c|}{ Mechanical Analysis } \\
\hline Sand & 67 & $\%$ \\
\hline Silt & 16 & $\%$ \\
\hline Clay & 17 & $\%$ \\
\hline Textural class & Sandy loam & \\
\hline $\mathrm{pH}(1: 1)$ & 7.55 & - \\
\hline EC $(1: 1$, water extract $)$ & 3.3 & $\mathrm{dS} / \mathrm{m}$ \\
\hline OM & 0.32 & $\%$ \\
\hline \multicolumn{3}{|c|}{ Soluble cations } \\
\hline $\mathrm{Ca}^{2+}$ & 9.52 & $\mathrm{meg} / \mathrm{l}$ \\
\hline $\mathrm{Mg}^{2+}$ & 8.16 & $\mathrm{meq} / \mathrm{l}$ \\
\hline $\mathrm{Na}^{+}$ & 11.76 & $\mathrm{meq} / \mathrm{l}$ \\
\hline $\mathrm{K}^{+}$ & 1.28 & $\mathrm{meq} / \mathrm{l}$ \\
\hline \multicolumn{3}{|c|}{ Soluble anions } \\
\hline $\mathrm{HCO}^{-}$ & 2.7 & $\mathrm{meq} / \mathrm{l}$ \\
\hline $\mathrm{Cl}^{-}$ & 7.5 & $\mathrm{meg} / \mathrm{l}$ \\
\hline $\mathrm{SO}_{4}^{2-}$ & 11.73 & $\mathrm{meq} / \mathrm{l}$ \\
\hline \multicolumn{3}{|c|}{ Available nutrients } \\
\hline Nitrogen (N) & 267.7 & $\mathrm{mg} / \mathrm{kg}$ \\
\hline Phosphorus (P) & 41.00 & $\mathrm{mg} / \mathrm{kg}$ \\
\hline Potassium (K) & 300.0 & $\mathrm{mg} / \mathrm{kg}$ \\
\hline
\end{tabular}

\section{RESULTS AND DISCUSSION}

\section{A) Growth parameters and yield}

Data in Tables (2 and 3) revealed that the fertilization treatment of $100 \%$ NPK gave, significantly, the highest plant height, number of braches/plant, fresh and dry weight $(\mathrm{g}) / \mathrm{plant}$, number of umbles, 100-seed weight, seed yield (g)/plant and seed yield (ton)/fed, while, the least growth parameters and yield was obtained from the control treatment during both seasons. It could be concluded that, the role of available mineral nutrition as essential elements in building coriander umbles due to the positive effect of NPK in increasing the vegetative growth photosynthetic, as well as, the increasing seed yield (ton)/fed. These results coincided with those obtained by El-Mahrouk (2000) on Swietteina mahogany seedling, reported that $120+240+120 \mathrm{~kg} \mathrm{NPK} / \mathrm{fed}$ and 24 I/day; gave the highest significant plant growth, also, Reshad (2002)recorded similar trendon coriander, too.

The obtained results given in Tables ( 2 and 3 )decleared that biofertilizers treatments exhibited a significant effect on all estimated traits during both seasons. Inoculation of mixture biofertilizer (phosphorein + cerealine + potassiomage) significantly increased growth parameters and yield. This finding could explain this positive effect of this mixture on growth characters and some yield in response to the phosphate potassium solubilizing bacteria and $\mathrm{N}$-fixing 
bacteria (cerealine) where this mixture may increase the synthesis of endogenous phytohormones i.e. indol acetic acid (IAA), gibberelline (GAS) and cytokinene(CKs) which play an important role in formation of a big active shoot and root systems which allow more nutrition uptake which reflect positively on both systems. The previous results agree, more or less, with the findings of Gad (2001) on Aneithum graveolens, Rashed (2002) on coriander, Abdel Latif (2002) on Cariumcarvi and Kandee let al. (2001) and Mohamed and Abdu (2004) on Foeniculumvulgare.

Tables (4 and 5) declared that the interaction between NPK fertilizer and biofertilization was significant on all growth parameters and yield. The superiority effect of applying $100 \%$ NPK plus interaction mixture of biofertilizerswas noticeable to plant height, number of braches/plant, fresh and dry weight,s (g)/plant, number of umbles, 100-seed weight, seed yield (g)/plant and seed yield (ton)/fed. 
Table (2).Plant height, number of branches, fresh and dry weight,sas affected by some macroelement,sand biofertilization during 2013 /2014 and2014 / 2015 seasons.

\begin{tabular}{|c|c|c|c|c|c|c|c|c|}
\hline \multirow{2}{*}{ Treatments } & \multicolumn{2}{|c|}{ Plant height (cm) } & \multicolumn{2}{|c|}{$\begin{array}{c}\text { Number of } \\
\text { branches/plant }\end{array}$} & \multicolumn{2}{|c|}{ Fresh weight/plant(g) } & \multicolumn{2}{|c|}{ Dry weight/plant (g) } \\
\hline & 2014 & 2015 & 2014 & 2015 & 2014 & 2015 & 2014 & 2015 \\
\hline \multicolumn{9}{|l|}{ A) NPKfertilizer } \\
\hline Control & $86.32 d$ & $92.33 c$ & $7.30 \mathrm{c}$ & $7.97 \mathrm{c}$ & $354.50 d$ & $385.42 c$ & $69.65 d$ & $79.43 d$ \\
\hline $50 \%$ NPK & $104.93 c$ & $112.75 b$ & $10.80 \mathrm{~b}$ & $12.00 \mathrm{~b}$ & $536.37 c$ & $681.41 \mathrm{a}$ & $103.38 c$ & $119.60 \mathrm{c}$ \\
\hline $75 \%$ NPK & $111.84 b$ & $119.79 a$ & $11.57 a$ & $12.87 c$ & $609.83 b$ & $657.7 \mathrm{~b}$ & $120.39 b$ & $131.18 b$ \\
\hline $100 \%$ NPK & $113.47 a$ & $121.28 a$ & $11.93 a$ & $13.20 \mathrm{a}$ & $634.51 \mathrm{a}$ & $681.83 a$ & $128.06 a$ & $138.14 a$ \\
\hline L.S.D.(0.05) & 1.10 & 1.05 & 0.50 & 0.60 & 10.70 & 12.30 & 2.90 & 3.20 \\
\hline \multicolumn{9}{|c|}{ B) Bio-fertilization } \\
\hline Uninoculation & $96.26 \mathrm{~d}$ & $104.07 d$ & $8.13 d$ & $9.25 d$ & $400.27 d$ & $448.25 e$ & $83.44 \mathrm{e}$ & $94.13 d$ \\
\hline Phosphorein & $103.32 c$ & $110.25 c$ & $9.12 \mathrm{c}$ & $10.79 c$ & $516.59 c$ & $584.19 c$ & $108.40 \mathrm{c}$ & $118.63 c$ \\
\hline Cerealine & $106.43 b$ & $114.61 b$ & $11.13 b$ & $12.33 b$ & $560.57 b$ & $605.58 b$ & $113.77 b$ & $123.88 b$ \\
\hline Potassmage & $105.95 b$ & $113.74 b$ & $9.25 c$ & $10.38 c$ & $527.16 c$ & $567.80 d$ & $104.88 d$ & $114.88 \mathrm{c}$ \\
\hline Mixed & $108.80 a$ & $117.38 a$ & $13.58 a$ & 14.92 & $627.80 a$ & $675.79 a$ & $124.77 a$ & $134.78 a$ \\
\hline L.S.D.(0.05) & 1.20 & 1.30 & 0.70 & 0.85 & 11.20 & 12.60 & 3.10 & 4.60 \\
\hline \multicolumn{9}{|l|}{ Interactions } \\
\hline$A \times B$ & * & * & * & * & * & * & * & * \\
\hline
\end{tabular}

Means of each factor designated by the same letter not significantly different at $5 \%$ using least Significant difference (L.S.D.)

*: Significant at 0.05 and 0.01 level of probability. 
Table (3).Number of umbles, 100-seed weight, seed yield plant and seed yield fed as affected by some macroelement,s and bio-fertilization during 2013 /2014 and 2014 / 2015 seasons.

\begin{tabular}{|c|c|c|c|c|c|c|c|c|}
\hline \multirow[t]{2}{*}{ Treatments } & \multicolumn{2}{|c|}{$\begin{array}{c}\text { Number of } \\
\text { umblers/plant }\end{array}$} & \multicolumn{2}{|c|}{100 -seed weight $(\mathrm{g})$} & \multicolumn{2}{|c|}{ Seed yield $(g) /$ plant } & \multicolumn{2}{|c|}{ Seed yield (ton)/fed } \\
\hline & 2014 & 2015 & 2014 & 2015 & 2014 & 2015 & 2014 & 2015 \\
\hline \multicolumn{9}{|l|}{ A) NPKfertilizer } \\
\hline Control & $104.43 d$ & $114.94 d$ & $1.45 \mathrm{c}$ & $1.46 \mathrm{c}$ & $73.40 c$ & $79.71 d$ & $0.880 d$ & $0.955 d$ \\
\hline $50 \%$ NPK & $157.07 c$ & $172.59 c$ & $1.55 b$ & $1.57 \mathrm{~b}$ & $82.51 b$ & $88.50 c$ & $0.967 c$ & $1.062 \mathrm{c}$ \\
\hline $75 \%$ NPK & $175.97 b$ & $191.84 b$ & $1.58 b$ & $1.61 b$ & $89.66 a$ & $94.30 \mathrm{~b}$ & $1.064 b$ & $1.135 b$ \\
\hline $100 \%$ NPK & $180.05 a$ & $197.83 a$ & $1.62 \mathrm{a}$ & $1.66 \mathrm{a}$ & $90.37 \mathrm{~b}$ & $96.54 \mathrm{a}$ & $1.085 \mathrm{a}$ & $1.158 \mathrm{a}$ \\
\hline L.S.D.(0.05) & 2.75 & 4.20 & 0.03 & 0.04 & 1.05 & 1.10 & 0.018 & 0.020 \\
\hline \multicolumn{9}{|c|}{ B) Bio-fertilization } \\
\hline Uninoculation & $133.23 \mathrm{e}$ & $134.79 d$ & $1.49 d$ & $1.51 d$ & $79.51 \mathrm{e}$ & $85.11 \mathrm{e}$ & $0.925 e$ & $1.021 \mathrm{e}$ \\
\hline Phosphorein & $144.59 c$ & $158.39 c$ & $1.52 \mathrm{c}$ & $1.55 c$ & $80.58 d$ & $86.70 d$ & $0.967 d$ & $1.040 \mathrm{~d}$ \\
\hline Cerealine & $164.66 b$ & $180.82 b$ & $1.56 b$ & $1.58 \mathrm{bc}$ & $83.66 \mathrm{c}$ & $89.07 \mathrm{c}$ & $1.004 \mathrm{c}$ & $1.071 \mathrm{c}$ \\
\hline Potassmage & $138.39 d$ & $153.57 \mathrm{c}$ & $1.58 a b$ & $1.61 \mathrm{ab}$ & $86.84 b$ & $91.88 b$ & $1.042 b$ & $1.102 b$ \\
\hline Mixed & $201.16 a$ & $218.97 a$ & $1.60 \mathrm{a}$ & $1.63 a$ & $88.12 a$ & $96.07 a$ & $1.057 \mathrm{a}$ & $1.151 \mathrm{a}$ \\
\hline L.S.D.(0.05) & 5.50 & 5.70 & 0.02 & 0.03 & 0.92 & 1.05 & 0.012 & 0.018 \\
\hline \multicolumn{9}{|l|}{ Interactions } \\
\hline Ax B & * & * & * & * & * & * & * & * \\
\hline
\end{tabular}

Means of each factor designated by the same letter not significantly different at $5 \%$ using least Significant difference (L.S.D.)

*: Significant at 0.05 and 0.01 level of probability. 
Table (4). Interaction between NPK fertilizer and biofertilization on fresh and dry weights/plant during $2013 / 2014$ and 2014 /2015 seasons.

\begin{tabular}{|c|c|c|c|c|c|}
\hline \multicolumn{2}{|c|}{ Treatments } & \multicolumn{2}{|c|}{ Fresh weight (g) } & \multicolumn{2}{|c|}{ Dry weight (g) } \\
\hline NPK fertilizer & Bio-fertilization & 2014 & 2015 & 2014 & 2015 \\
\hline \multirow{5}{*}{ Control } & Uninoculation & 311.20 & 343.44 & 57.87 & 67.33 \\
\hline & Phosphorein & 354.47 & $381 . .64$ & 71.17 & 81.50 \\
\hline & Cerealine & 362.10 & 393.05 & 73.00 & 83.00 \\
\hline & Potassmage & 339.47 & 366.90 & 67.40 & 76.60 \\
\hline & Mixed & 405.33 & 439.06 & 78.83 & 88.70 \\
\hline \multirow{5}{*}{$50 \%$ NPK } & Uninoculation & 354.40 & 392.02 & 79.57 & 89.60 \\
\hline & Phosphorein & 352.53 & 597.07 & 113.43 & 123.40 \\
\hline & Cerealine & 568.13 & 615.64 & 116.00 & 127.00 \\
\hline & Potassmage & 562.40 & 606.23 & 111.23 & 122.20 \\
\hline & Mixed & 644.37 & 697.57 & 126.67 & 136.70 \\
\hline \multirow{5}{*}{ 75\% NPK } & Uninoculation & 473.87 & 513.23 & 95.17 & 105.20 \\
\hline & Phosphorein & 614.93 & 644.44 & 119.83 & 129.90 \\
\hline & Cerealine & 653.87 & 706.18 & 133.40 & 143.50 \\
\hline & Potassmage & 592.67 & 636.50 & 116.10 & 126.40 \\
\hline & Mixed & 708.80 & 768.13 & 140.47 & 150.60 \\
\hline \multirow{6}{*}{$100 \%$ NPK } & Uninoculation & 501.60 & 544.31 & 101.13 & 111.40 \\
\hline & Phosphorein & 644.27 & 693.60 & 129.17 & 139.20 \\
\hline & Cerealine & 658.14 & 707.46 & 132.67 & 142.70 \\
\hline & Potassmage & 614.13 & 661.55 & 124.20 & 154.30 \\
\hline & Mixed & 754.40 & 798.39 & 153.10 & 163.10 \\
\hline & 5) & 11.80 & 12.20 & 3.70 & 4.90 \\
\hline
\end{tabular}

Table (5). Interaction between NPK fertilizer and biofertilization on 100seed weight and seed yield during 2013/2014 and2014 / 2015 seasons.

\begin{tabular}{cccccc}
\hline \multicolumn{2}{c}{ Treatments } & \multicolumn{2}{c}{ 100-seed weight (g) } & \multicolumn{2}{c}{ Seed yield (g)/plant } \\
\hline \multirow{2}{*}{ NPK fertilizer } & $\begin{array}{c}\text { Bio- } \\
\text { fertilization }\end{array}$ & $\mathbf{2 0 1 3 / 2 0 1 4}$ & $\mathbf{2 0 1 4 / 2 0 1 5}$ & $\mathbf{2 0 1 3 / 2 0 1 4}$ & $\mathbf{2 0 1 4 / 2 0 1 5}$ \\
\hline & Uninoculation & 1.40 & 1.42 & 70.31 & 75.73 \\
Control & Phosphorein & 1.44 & 1.45 & 70.55 & 77.03 \\
& Cerealine & 1.47 & 1.47 & 75.12 & 80.43 \\
& Potassmage & 1.47 & 1.48 & 76.88 & 82.07 \\
& Mixed & 1.48 & 1.50 & 73.83 & 83.30 \\
\hline & Uninoculation & 1.50 & 1.50 & 76.47 & 83.44 \\
\multirow{5}{*}{$50 \%$ NPK } & Phosphorein & 1.51 & 1.53 & 77.84 & 83.98 \\
& Cerealine & 1.57 & 1.58 & 80.39 & 86.84 \\
& Potassmage & 1.59 & 1.61 & 87.33 & 91.74 \\
& Mixed & 1.56 & 1.63 & 90.51 & 96.48 \\
\hline & Uninoculation & 1.51 & 1.5 & 84.62 & 89.39 \\
$75 \%$ NPK & Phosphorein & 1.55 & 1.58 & 85.63 & 91.44 \\
& Cerealine & 1.58 & 1.63 & 88.04 & 93.76 \\
& Potassmage & 1.60 & 1.65 & 91.18 & 96.56 \\
& Mixed & 1.65 & 1.66 & 92.83 & 100.35 \\
\hline & Uninoculation & 1.55 & 1.59 & 86.63 & 91.89 \\
& Phosphorein & 1.58 & 1.62 & 88.01 & 94.33 \\
& Cerealine & 1.60 & 1.65 & 91.10 & 95.23 \\
$100 \%$ NPK & Potassmage & 1.66 & 1.69 & 91.83 & 97.14 \\
& Mixed & 1.70 & 1.73 & 94.30 & 104.10 \\
\hline & & $\mathbf{0 . 0 3}$ & $\mathbf{0 . 0 4}$ & $\mathbf{1 . 3 0}$ & $\mathbf{1 . 4 5}$ \\
\hline
\end{tabular}




\section{B) Total chlorophyll and Chemical composition}

Data in Table (6) showed the effect of NPKfertilizer and biofertilization on total chlorophyll, $\mathrm{N} \%, \mathrm{P} \%$ and $\mathrm{K} \%$ in the leaves during the bothseasons.As for the effect of NPK fertilization obtained data showed that the treatment of $100 \% \mathrm{NPK}$, gavethe highest total chlorophyll (1.864 and 1.378 (mg/g F.W.)N ( 2.29 and 2.33\%), P (0.599 and $1.592 \%)$ andK(5.19 and $5.69 \%)$,for the both seasons respectively while theleasttotal chlorophyll, and $\mathrm{N}, \mathrm{P}$ and $\mathrm{K} \%$ were obtained from the control treatment during bothseasons. Results in Table (6) declared that thetotal chlorophylland chemical compositionincreased due to using all different commercial biofertilizers when compared to the uninoculation (control) treatment with significant differences in the most traits during both season. However, the highest mean values of chemical composition during both seasons were observed due to inoculation by the mixture biofertilizer. On the other hand, bio-fertilizers treatment resulted in the highest values of all studied parameters in comparison to the other treatments during both seasons. Hence, it could be concluded that these findings may be taken place due to that the active role of phosphorus, potassium dissolving bacteria and $\mathrm{N}$-fixation (cerealine) and increasing the endogenous phytohormones as reported earlier which play an important rolesin life cycles of both shoot and root systems.

These obtained results are in compatible with those obtained by Kandeel et al. (2001) on Foeniculum vulgare and Osman (2000) on coriander. The interaction between NPKf ertilizer and biofertilization was significant on alltraits during both seasons (Table 7), whereas, theapplication of $100 \%$ NPK and mixturebiofertilization on root zone of plant as result of adding fertilization treatments reflected positively on nutrients uptake by plants and confirm the previous vegetative growth. Similar results, more or less, were obtained by Aly (1994) on saponaria. Also, Jacoub (1999) on Ocimum basilicum, found that as all NPK rates increased; chemical compositionin the leaves and stem increased. 
Table (6).Total chl. and $\mathrm{N}, \mathrm{P}$ and $\mathrm{K} \%$ as affected by some macroelements and bio-fertilization during during 2013/2014 and 2014 / 2015 seasons.

\begin{tabular}{|c|c|c|c|c|c|c|c|c|}
\hline \multirow[t]{2}{*}{ Treatments } & \multicolumn{2}{|c|}{$\begin{array}{c}\text { Total chlorophyll } \\
(\mathrm{mg} / \mathrm{g})\end{array}$} & \multicolumn{2}{|c|}{ N\% } & \multicolumn{2}{|c|}{$\mathrm{P} \%$} & \multicolumn{2}{|c|}{$\mathrm{K} \%$} \\
\hline & 2014 & 2015 & 2014 & 2015 & 2014 & 2015 & 2014 & 2015 \\
\hline \multicolumn{9}{|l|}{ A) NPKfertilizer } \\
\hline Control & $0.875 d$ & $0.884 d$ & $1.16 d$ & $1.21 d$ & $0.334 d$ & $0.352 d$ & $2.36 d$ & $3.05 d$ \\
\hline $50 \%$ NPK & $1.069 c$ & $1.079 c$ & $1.47 \mathrm{c}$ & $1.49 c$ & $0.478 c$ & $0.465 c$ & $3.53 c$ & $3.91 c$ \\
\hline $75 \%$ NPK & $1.195 b$ & $1.207 b$ & $1.83 b$ & $1.88 b$ & $0.537 b$ & $0.531 b$ & $4.35 b$ & $4.81 b$ \\
\hline $100 \%$ NPK & $1.364 a$ & $1.378 a$ & $2.29 a$ & $2.33 a$ & $0.599 a$ & $0.592 a$ & $5.19 a$ & $5.69 a$ \\
\hline L.S.D.(0.05) & 0.080 & 0.087 & 0.20 & 0.25 & 0.055 & 0.057 & 0.45 & 0.40 \\
\hline \multicolumn{9}{|c|}{ B) Bio-fertilization } \\
\hline Uninoculation & $1.017 d$ & $1.026 \mathrm{~d}$ & $1.44 d$ & $1.49 d$ & $0.402 d$ & $0.335 d$ & $3.52 d$ & $3.85 c$ \\
\hline Phosphorein & $1.106 c$ & $1.117 c$ & $1.67 \mathrm{c}$ & $1.69 c$ & $0.508 b$ & $0.542 b$ & $3.74 \mathrm{~cd}$ & $4.13 b$ \\
\hline Cerealine & $1.176 b$ & $1.188 b$ & $1.80 b$ & $1.83 b$ & $0.485 c$ & $0.501 b$ & 3.99b & $4.28 b$ \\
\hline Potassmage & $1.076 \mathrm{c}$ & $1.086 c$ & $1.67 \mathrm{c}$ & $1.70 c$ & $0.445 c$ & $0.451 \mathrm{c}$ & $4.29 a$ & $4.71 \mathrm{a}$ \\
\hline Mixed & $1.256 a$ & $1.267 a$ & $1.89 a$ & $1.92 a$ & $0.582 a$ & $0.583 a$ & $4.35 a$ & $4.84 a$ \\
\hline L.S.D.(0.05) & 0.075 & 0.072 & 0.07 & 0.07 & 0.060 & 0.065 & 0.22 & 0.19 \\
\hline \multicolumn{9}{|l|}{ Interactions } \\
\hline Ax B & * & * & * & * & * & * & * & * \\
\hline
\end{tabular}


Table (7).Total chl. and N, P, and K\% as effected by Interaction between NPK fertilizer and bio-fertilizer sources during 2013 / 2014 and 2014 /2015 seasons.

\begin{tabular}{|c|c|c|c|c|c|c|c|c|c|}
\hline \multicolumn{2}{|c|}{ Treatments } & \multicolumn{2}{|c|}{$\begin{array}{l}\text { Total Chlorophyll } \\
\text { (mg/g F.W.) }\end{array}$} & \multicolumn{2}{|c|}{$\mathbf{N}(\%)$} & \multicolumn{2}{|c|}{ P (\%) } & \multicolumn{2}{|c|}{ K (\%) } \\
\hline $\begin{array}{c}\text { NPK } \\
\text { fertilizer }\end{array}$ & Bio-fertilization & 2013/2014 & 2014/2015 & 2013/2014 & 2014/2015 & 2013/2014 & 2014/2015 & $2013 / 2014$ & $2014 / 2015$ \\
\hline \multirow{5}{*}{ Control } & Uninoculation & 0.765 & 0.772 & 1.00 & 1.08 & 0.247 & 0.267 & 2.30 & 2.50 \\
\hline & Phosphorein & 0.852 & 0.861 & 1.14 & 1.16 & 0.354 & 0.401 & 2.59 & 2.94 \\
\hline & Cerealine & 0.903 & 0.912 & 1.23 & 1.28 & 0.350 & 0.344 & 2.81 & 3.09 \\
\hline & Potassmage & 0.827 & 0.837 & 1.21 & 1.21 & 0.317 & 0.340 & 3.00 & 3.30 \\
\hline & Mixed & 1.028 & 1.034 & 1.24 & 1.29 & 0.400 & 0.407 & 3.10 & 3.40 \\
\hline \multirow{5}{*}{ NPK } & Uninoculation & 0.962 & $0 . .972$ & 1.29 & 1.22 & 0.384 & 0.307 & 3.17 & 3.49 \\
\hline & Phosphorein & 1.069 & 1.076 & 1.44 & 1.47 & 0.504 & 0.534 & 3.23 & 3.57 \\
\hline & Cerealine & 1.134 & 1.147 & 1.54 & 1.57 & 0.477 & 0.490 & 3.36 & 3.68 \\
\hline & Potassmage & 1.035 & 1.046 & 1.50 & 1.53 & 0.447 & 0.440 & 3.92 & 4.34 \\
\hline & Mixed & 1.140 & 1.152 & 1.60 & 1.64 & 0.574 & 0.551 & 3.99 & 4.48 \\
\hline \multirow{5}{*}{$\begin{array}{l}75 \% \\
\text { NPK }\end{array}$} & Uninoculation & 1.108 & 1.118 & 1.56 & 1.62 & 0.480 & 0.354 & 3.82 & 4.18 \\
\hline & Phosphorein & 1.183 & 1.197 & 1.81 & 1.85 & 0.544 & 0.587 & 4.09 & 4.48 \\
\hline & Cerealine & 1.263 & 1.276 & 1.93 & 1.98 & 0.520 & 0.540 & 4.18 & 4.59 \\
\hline & Potassmage & 1.153 & 1.161 & 1.72 & 1.80 & 0.494 & 0.474 & 4.81 & 5.35 \\
\hline & Mixed & 1.266 & 1.282 & 2.12 & 2.18 & 0.644 & 0.647 & 4.83 & 5.42 \\
\hline \multirow{5}{*}{$\begin{array}{l}100 \% \\
\text { NPK }\end{array}$} & Uninoculation & 1.231 & 1.242 & 1.92 & 2.00 & 0.497 & 0.410 & 4.79 & 5.23 \\
\hline & Phosphorein & 1.319 & 1.332 & 2.27 & 2.29 & 0.627 & 0.644 & 5.01 & 5.53 \\
\hline & Cerealine & 1.401 & 1.417 & 2.46 & 2.48 & 0.590 & 0.627 & 5.27 & 5.74 \\
\hline & Potassmage & 1.287 & 1.298 & 2.23 & 2.27 & 0.520 & 0.550 & 5.39 & 5.86 \\
\hline & Mixed & 1.587 & 1.599 & 2.56 & 2.58 & 0.707 & 0.727 & 5.47 & 6.07 \\
\hline \multicolumn{2}{|c|}{ L.S.D.(0.05) } & 0.084 & 0.090 & 0.29 & 0.30 & 0.064 & 0.068 & 0.48 & 0.43 \\
\hline
\end{tabular}




\section{C) Major components percentage of essential oil}

The effect of NPK fertilization treatments and biofertilization on essential oil of major components (Linalool, a-2-deenol, a- Pinene, Camphor and Geraniol) percentages are shown in Table (8). The recorded results indicated that using application $75 \%$ NPKfertilization treatments had thehighest values for the studiedmajor components percentage of coriander oil in the sameTable. The interaction with potassiomage and mixture biofertilizer, brought about gave the highest percentage of major components in 2014 / 2015season.Similarresults were reported by Darzi et al. (2011) on anisum and Khalil (2008) on Foeniculum vulgare.

The highest values were produced by applied at $75 \%$ NPK with inoculation of potassmage and mixed biofertilizer (Table8).

Table (8). Major components percentage as affected by mineral and biofertilization during $2014 / 2015$ seasons.

\begin{tabular}{|c|c|c|c|c|c|}
\hline Treatments & Linalool\% & $\alpha-2-$ deenol\% & $\alpha-P i n e n e \%$ & Camphor\% & Geraniol\% \\
\hline \multicolumn{6}{|l|}{ A) NPK fertilizer } \\
\hline Control & $47.08 d$ & $13.47 d$ & $432 c$ & $5.19 c$ & 1.95 \\
\hline $50 \%$ NPK & $48.31 b$ & $15.00 \mathrm{~b}$ & $4.53 b$ & $5.54 b$ & 2.34 \\
\hline $75 \%$ NPK & $48.38 a$ & $15.10 \mathrm{a}$ & $4.61 \mathrm{a}$ & $5.87 a$ & 2.70 \\
\hline $100 \%$ NPK & $48.01 \mathrm{e}$ & $14.89 \mathrm{c}$ & $4.59 a$ & $5.88 a$ & 2.53 \\
\hline L.S.D.(0.05) & 0.05 & 0.06 & 0.04 & 0.06 & 0.06 \\
\hline \multicolumn{6}{|c|}{ B) Bio-fertilization } \\
\hline Uninoculation & $47.95 d$ & $14.58 \mathrm{~b}$ & $4.49 c$ & $5.46 d$ & 2.20 \\
\hline Phosphorein & $48.06 \mathrm{c}$ & $14.56 \mathrm{~b}$ & $4.50 \mathrm{bc}$ & $5.57 c$ & $2.35 b$ \\
\hline Cerealine & $48.11 b c$ & $14.64 a$ & $4.52 \mathrm{ab}$ & $5.64 b$ & 2.38 \\
\hline Potassmage & $48.32 a$ & $14.65 a$ & $4.53 a$ & $5.69 a b$ & $2.50 a$ \\
\hline Mixed & $48.14 b$ & $14.67 a$ & $4.54 a$ & $5.74 \mathrm{a}$ & $2.49 a$ \\
\hline L.S.D.(0.05) & 0.06 & 0.05 & 0.02 & 0.05 & 0.04 \\
\hline \multicolumn{6}{|l|}{ Interactions } \\
\hline Ax B & * & * & * & * & * \\
\hline
\end{tabular}


Table (9). Interaction between mineral fertilizer and bio-fertilization on major components percentage in 2014 / 2015 seasons.

\begin{tabular}{ccccccc}
\hline $\begin{array}{c}\text { NPK } \\
\text { fertilizer }\end{array}$ & $\begin{array}{c}\text { Bio- } \\
\text { fertilization }\end{array}$ & Linolool & a-2-deenol & a-Pinene & camphor & Geraniol \\
\hline & Uninoculation & 47.08 & 13.46 & 4.30 & 5.02 & 1.85 \\
Control & Phosphorein & 47.11 & 13.47 & 4.30 & 5.11 & 1.94 \\
& Cerealine & 47.30 & 13.47 & 4.33 & 5.23 & 1.96 \\
& Potassmage & 47.20 & 13.48 & 4.34 & 5.31 & 2.01 \\
& Mixed & 47.50 & 13.47 & 4.33 & 5.30 & 2.00 \\
\hline \multirow{2}{*}{$50 \%$} & Uninoculation & 48.31 & 14.82 & 4.48 & 5.38 & 1.98 \\
NPK & Phosphorein & 48.42 & 14.91 & 4.49 & 5.51 & 2.31 \\
& Cerealine & 48.48 & 15.02 & 4.53 & 5.51 & 2.38 \\
& Potassmage & 48.91 & 15.08 & 4.56 & 5.59 & 2.52 \\
& Mixed & 48.75 & 15.18 & 4.61 & 5.62 & 2.52 \\
\hline & Uninoculation & 48.38 & 15.11 & 4.59 & 5.63 & 2.45 \\
$75 \%$ & Phosphorein & 48.41 & 15.03 & 4.61 & 5.73 & 2.60 \\
NPK & Cerealine & 48.51 & 15.15 & 4.61 & 5.92 & 2.63 \\
& Potassmage & 48.83 & 15.11 & 4.63 & 5.95 & 2.91 \\
& Mixed & 48.61 & 15.11 & 4.62 & 6.14 & 2.89 \\
\hline \multirow{2}{*}{$100 \%$} & Uninoculation & 48.01 & 14.91 & 4.59 & 5.81 & 2.51 \\
NPK & Phosphorein & 48.31 & 14.82 & 4.59 & 5.92 & 2.53 \\
& Cerealine & 48.32 & 14.89 & 4.60 & 5.91 & 2.53 \\
& Potassmage & 48.31 & 14.91 & 4.60 & 5.90 & 2.54 \\
& Mixed & 48.31 & 14.92 & 4.59 & 5.88 & 2.56 \\
\hline L.S.D.(0.05) & $\mathbf{0 . 0 5}$ & $\mathbf{0 . 0 6}$ & $\mathbf{0 . 0 4}$ & $\mathbf{0 . 0 5}$ & $\mathbf{0 . 0 5}$ \\
\hline
\end{tabular}

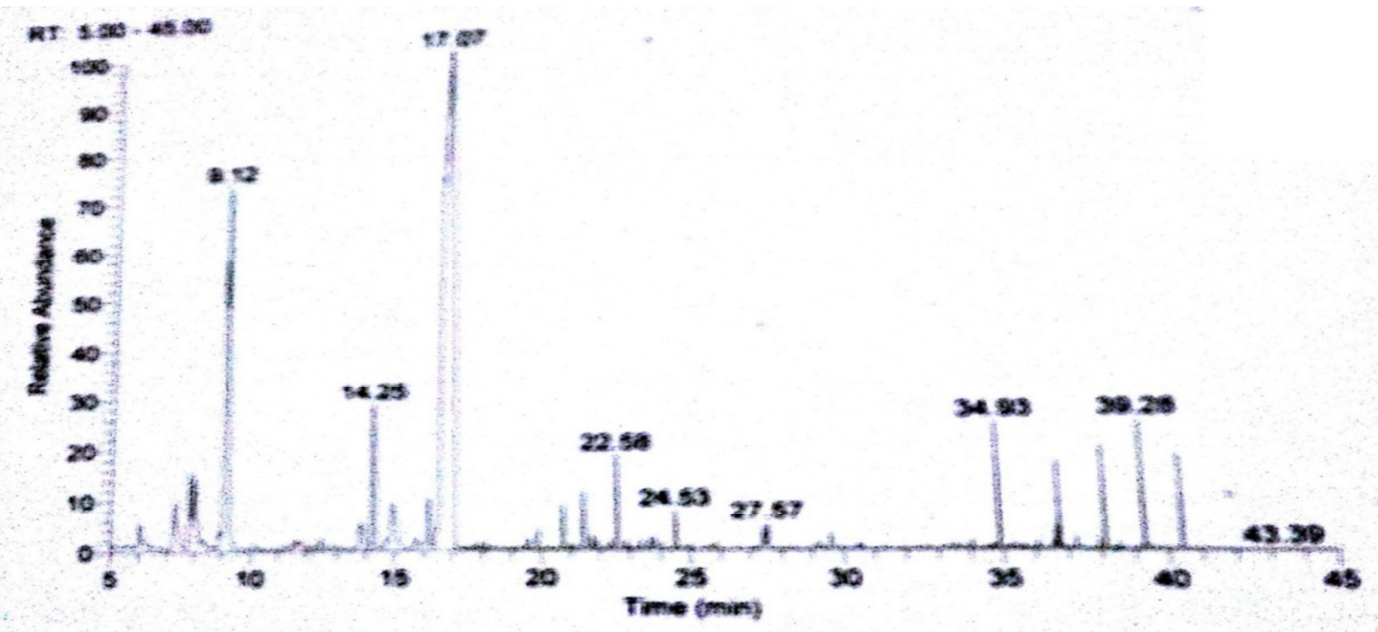

Fig (1). Typical chromatogram of Coriander oil 


\section{REFERENCES}

Abdel Latif, T. A. (2002). Effect of organic manure and biofertilizer on caraway plants (Carumcarvi, L.) Agric. Sci. Mansoura Univ., 27(5): 3459-3468.

Aly, A.S. (1994). Effect of chemical fertilization and growth regulators on growth, yield and active ingredients of soapwort plant (Saponaria officinalis, L.). Ph. D. Thesis, Fac. Agric. Cairo Univ.

British Pharmacopoeia (1963). Determination of Volatile Oils in Drugs. The Pharmaceutical Press, 17 Bloomsbury Square, London, WC1.

Cantwell, M. T. and M. S. Reid (1993). Postharvest physiological and handling of fresh culinary herbs. J. Herbs species. Med. PI. 1:93-127.

Chapman, H. D. and P.F. Pratt (1978). Method of Analysis for Soil and Water. 2nd Ed., Chapter, 17:150-161. Uni. Calif. Div. Agric. Sci. USA.

Darzi, M. T., M. R. Hay SeyedHadi and F. Rejall (2011). Effect of vermicompost and phosphate biofertilizers application on yield and yield components in anise (Pimpinella ansisum, L.). Iran J. Med. Aroma plants, 4(50): 451-465.

El-Mahrouk, E. M. (2000). Response of Swietteniamahogoni, L. Jog to different levels of irrigation water and NPK fertilization treatments in a new, by reclaimed area. J. Agric. Re. Tanta Univ., 26(2): 377-390.

Gad, W.M. (2001). Physiological studies on FoeniculumvulgareMill. andAnethumgraveolensL. M.Sc. Thesis. Faculty Agric., Kafr El-Sheikh, Tanta Univ., Egypt.

Gomez, K. A. and A. A. Gomez (1984). Statistical Procedure for Agricultural Research, Jhon Willey and Sons. Inc. New York.

Gunther, Z., and S. Joseph (1978). Hand Book Series in Chromatography. CRC press, Inc.

Heftman, E. (1967). Chromatography. Reinhold Pub. Crop. New York

Hussein, M. S. (1995). Response of growth, yield and essential oil of coriander and dill to different nitrogen sources. Egypt. J. Hort. Sci., 22 (1): 1-10.

Jackson, M.L. (1973). Soil chemical analysis, Prentice Hall of India private limited, New Delhi, P. 498.

Jacoub, R. W. (1999). Effect of some organic and inorganic fertilizers on growth, oil yield and chemical composition of Ocimum basilicum L. and Thymus vulgaris L. plants,. PhD. Thesis, Fac. Agric., Cairo Uni., Egypt.

Kandeel, Y.R. , E.S. Nofal, F.A. Menesi, K.A. Reda, M. Taher and Z.T. Zaki (2001). Effect of some cultural practices on growth and chemical composition of Foeniculum vulgare Mill. Proc. Fifth. Arab. Hort. Conf. Ismailia, Egypt. pp. 61-72.

Khalil, M. Y., A.M. Kandil and M.F. SwaefyHend (2008). Effect of three different compost levels on fennel and salvia growth characters and their essential oils. Res. J. Agric. \&Biol. Sci., 4(1): 34-39.

Lowther, G.R. (1980). Using of a single $\mathrm{H}_{2} \mathrm{So}_{4}-\mathrm{H}_{2} \mathrm{O}_{2}$ digest for the analysis of Pinus radiate needles. Commun.Soil Sci. PI. Analysis, 11: 175-188.

Mohamed, M.A.H. and M. Abdu (2004). Growth and oil production of fennel (Foeniculum vulgare Mill): Effect of irrigation and organic fertilization. Biological Agric. \& Hort., 22: 31-39.

Moran, M.J. (1982). Availability Analysis: A Guide to Efficient EnergyUse, Prentice Hall NJ USA. 
Osman, Y.A.H. (2000). Possibility of production of coriander (CoriandrumsativumL) under Sinai conditions. Ph.D. Thesis, Fac, Agric, Cairo Univ.

Page, A.L., R.H. Miller and D.R. Keeny (1982).Methods of soil analysis part 2 Amer. Soc. Agric. Inc. Madison W19:595.

Rashed, N. M. M. (2002). Effect of fertilization on the growth and storability of some aromatic plants. M. SC. Thesis Fac. Agric Kafr El-Sheikh, Tanta Univ. Egypt.

$$
\text { الملخص العربي }
$$

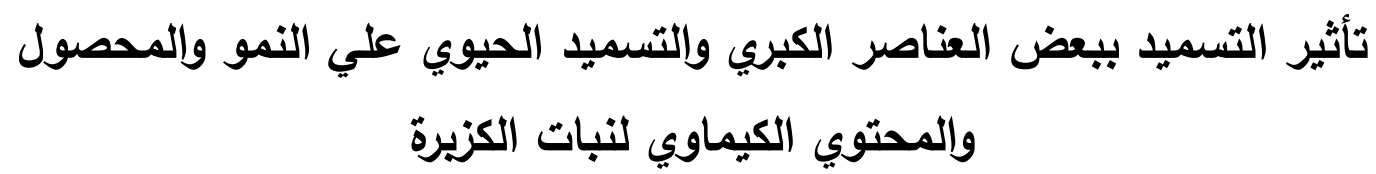
وائل محمد إسماعيل نصر الله *فتحي إبراهيم راضوان *علي إبراهيم علي عبيدو

\section{* السيد حسين شعبان *}

"قسم إلانتاج النباتي - كلية الزراعة سابا باثا - جامعة الإسكندرية

**قمم بحوث النباتات الطبية والعطرية - مركز البحوث الزراعية - شعبة إنتاج وتكنولوجيا

النباتات الطبية والعطرية

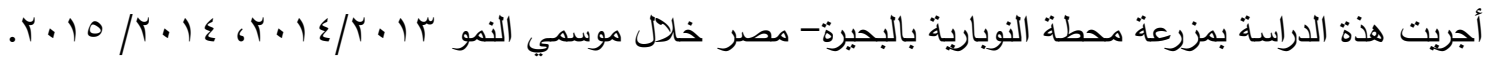
وكان الهدف من هذه الدراسة هو دراسة تأثير التسميد المعدني والحيوي علي النمو الخضري والإنتاج والمكون الكيماوي والزيوت الفعالة وكذلك المكونات الرئيسية لنمو النبات ولذلك أقيمت تجربتان حقليتان لاراسة تأثنير التسميد المعدني والحيوي علي النمو الخضري، المحصول، المكونات الكيميائية شاملة محتوي الزيت بالإضافة الي محتوي المكونات الفعالة للزيت لنبات الكزبرة. حيث صمدت التجربة بتصميم القطع المنشقة مرة واحدة مع ثلاث مكرارت.

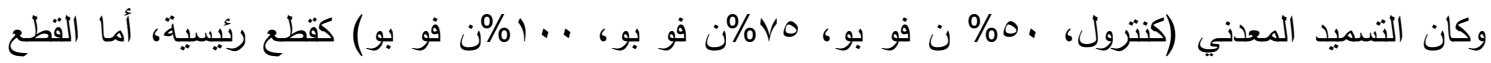
الثقية فوزعت بخمس معاملات تسميد حيوي (بدون تلقيح، فوسفورين، سيريالين، بوتاسيوماج، خليط الأسمدة • الحيوبة)

\section{ويمكن تلخيص أهم النتائج المتحصل عليها فيما يلي:}

ا. كان لإضافة التسميد المعني عند . . (\% ن فو بو تأثنراً معنوياً علي صفات النمو الخضري، المحصول،

$$
\text { المكونات الكيميائية مقارنة بالكنترول لنباتات الكزبرة في كلا الموسمين. }
$$

r. كان أفضل تداخل بين إضافة . . (\% ن فو بومع خليط الأسمدة الحيوية (فوسفورين، سيريالين، بوناسيوماج)

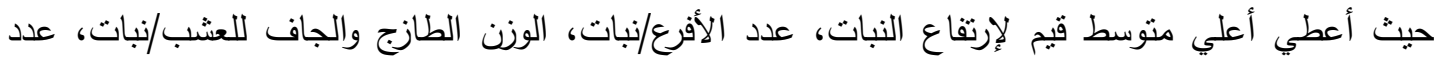
النورات/نبات، وزن . 1 بذرة، محصول البذور/فدان إلي النسبة المئوية للزيت ولكن محتوي مكونات الزيت الزيت

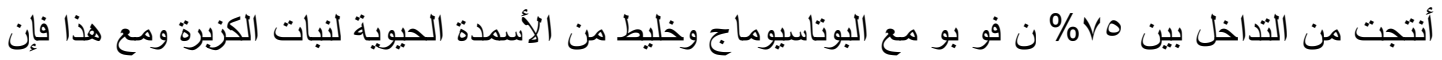


الصفات تحت الدراسة زادت معنوياً عند معاملات التلقيح مقارنة بدون تلقيح (الكنترول) حيث أن خليط الأسمدة الحيوية كان أفضل معاملة .

r. هذه الدراسة تقترح مزيد من الدراسة لتأثثر معدلات التسميد المعدني والحيوي علي نبات الكزبرة تحت مختلف

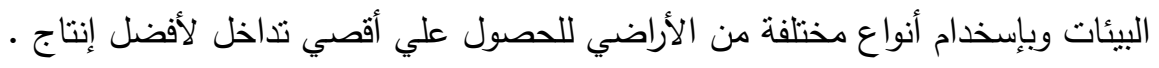

